\title{
Dirac $b$ quark on the lattice
}

\section{TWQCD Collaboration: Ting-Wai Chiu ${ }^{a}$, Tung-Han Hsieh ${ }^{* b}$, Chao-Hsi Huang ${ }^{c}$, and Kenji Ogawa ${ }^{d}$}

${ }^{a}$ Department of Physics, Center for Theoretical Sciences, and National Center for Theoretical Sciences, National Taiwan University, Taipei 10617, Taiwan.

Email: twchiulphys.ntu.edu.tw

${ }^{b}$ Research Center for Applied Sciences, Academia Sinica, Taipei 115, Taiwan.

Email: thhsieh@twcp1.phys.ntu.edu.tw

${ }^{c}$ Department of Physics, National Taiwan University, Taipei 10617, Taiwan.

Email: chao@twcpl.phys.ntu.edu.tw

${ }^{d}$ Department of Physics, National Taiwan University, Taipei 10617, Taiwan.

Email: ogawak@phys.ntu.edu.tw

We perform the first study of treating $\mathbf{b}, \mathbf{c}$, and $\mathbf{s}$ quarks as Dirac fermions in lattice QCD with exact chiral symmetry. On a $32^{3} \times 60$ lattice with $a^{-1} \simeq 7.68 \mathrm{GeV}$, we compute point-to-point quark propagators, and measure the time-correlation functions for mesons with quark contents $\mathbf{b} \overline{\mathbf{b}}, \mathbf{c} \overline{\mathbf{b}}, \mathbf{s} \overline{\mathbf{b}}$, and $\mathbf{c} \overline{\mathbf{c}}$. The lowest-lying meson mass spectra, the pseudoscalar decay constants, and the $\mathbf{b}$ and $\mathbf{c}$ quark masses are determined.

The XXV International Symposium on Lattice Field Theory

July 30-4 August 2007

Regensburg, Germany

${ }^{*}$ Speaker. 


\section{Introduction}

Spectroscopy with heavy quark is one of the major topics in high energy physics. Theoretically, since the b quark is very heavy $(\sim 4.6 \mathrm{GeV})$, most theoretical approaches regard the b quark as a static, non-relativistic particle, or treat it with heavy quark effective theory or the relativistic heavy quark formalism. In other words, the heavy $\mathbf{b}$ quark is not treated as the Dirac fermion. This introduces complicated normalization procedures, in which the systematic errors are difficult to control.

In QCD, all quarks are excitations of Dirac fermion fields, thus it is vital to preserve this important feature in any theoretical study. However, so far it remains a challenge for a single lattice to accommodate such a wide range of quark masses from $m_{u / d}$ quark to $m_{b}$. If we restrcit our study to the hadrons containing only $\mathbf{s}$, $\mathbf{c}$, and $\mathbf{b}$ valence quarks (ranging from $140 \mathrm{MeV}$ to 4.6 $\mathrm{GeV}$ ) satisfying the constraints $m_{q} a<1$ and $M_{h} L>4$, then it is possible to accommodate these hadrons on a $32^{3} \times 60$ lattice, with the inverse of lattice spacing $a^{-1} \simeq 7.68 \mathrm{GeV}$.

In this proceeding, we report the first study of heavy meson spectra [1], by treating all heavy and light quarks as Dirac fermions, in lattice QCD with exact chiral symmetry. Namely, we restrict ourselves to mesons with quark contents $\mathbf{b} \overline{\mathbf{b}}, \mathbf{c} \overline{\mathbf{b}}, \mathbf{s} \overline{\mathbf{b}}$, and $\mathbf{c} \overline{\mathbf{c}}$. Our results of masses and decay constants of the pseudoscalar mesons $B_{s}$ and $B_{c}$, and the masses of the vector mesons $B_{s}^{*}$ and $B_{c}^{*}$, have been presented in [2].

We adopt the optimal domain-wall fermion (ODWF) proposed by Chiu [3, 4] as our fermion scheme, which possesses optimal chiral symmetry for any finite $N_{s}$ (the number of sites in the 5-th dimension), and also preserves the salient features of the Dirac fermion in the continuum. With 100 gauge configurations generated with the single plaquette action at $\beta=7.2$, we compute the pointto-point quark propagators for 33 quark masses in the range $0.01 \leq m_{q} a \leq 0.85$. We set the fifth dimension of ODWF, $N_{s}$, to 128 , and the stopping criteria of the conjugate gradient (CG) iterations to $10^{-11}$, such that the chiral symmetry breaking $\sigma$ and the residual of the quark propagators $\varepsilon$ satisfy

$$
\sigma=\left|Y^{\dagger} S^{2} Y\right| /\left|Y^{\dagger} Y\right|<10^{-14}, \quad \varepsilon=\left\|\left(D_{c}+m_{q}\right) Y-\mathbb{I}\right\|<2 \times 10^{-11}
$$

where $S$ is the optimal rational approximation of the sign function of $\gamma_{5} D_{w}, D_{w}$ is the Wilson Dirac operator plus a negative parameter $-m_{0}$. Here $\left(D_{c}+m_{q}\right)^{-1}$ is the valence quark propagator, and $Y$ is the solution vector of solving the quark propagators via the $\mathrm{CG}$ algorithm. Note that our massive Dirac operator is exponentially local for all the quark masses. In Fig. 1, we plot the magnitude of the 4-D effective Dirac operator of ODWF, $D_{\text {eff }}(x ; 0)$ (i.e., the overlap-Dirac operator with Zolotarev approximation of the sign function) [4] along the t-axis, for one gauge configuration. Evidently, it is exponentially-local. Furthermore, we have checked that for any element of $D_{\mathrm{eff}}^{a \alpha ; b \beta}(x ; 0)$ (where $a, b=1,2,3$ and $\alpha, \beta=1,2,3,4)$, its magnitude is also exponentially-local along any direction, for all gauge configurations and all 33 quark masses.

Next, we measure the time-correlation function

$$
C_{\Gamma}(t)=\left\langle\sum_{\vec{x}} \operatorname{tr}\left\{\Gamma\left(D_{c}+m_{Q}\right)_{x, 0}^{-1} \Gamma\left(D_{c}+m_{q}\right)_{0, x}^{-1}\right\}\right\rangle
$$




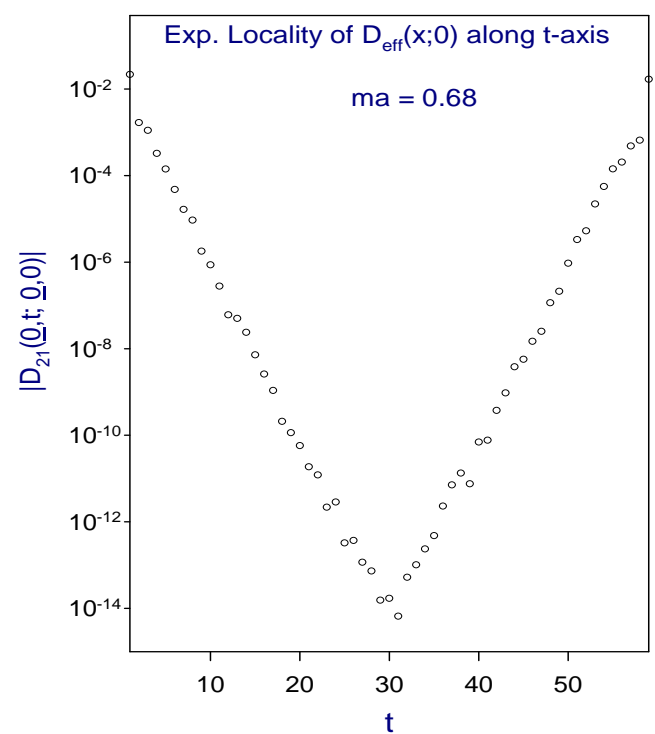

(a)

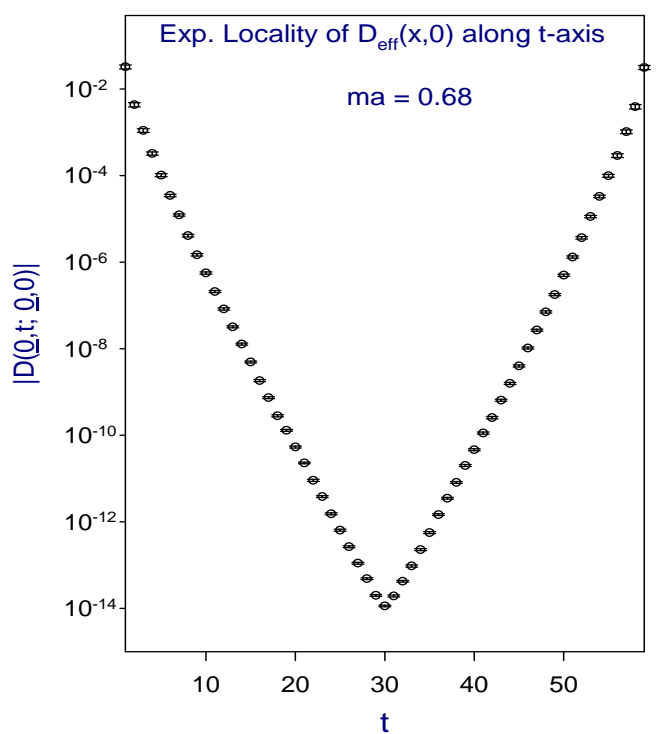

(b)

Figure 1: (a) The magnitude of the an element of $D_{\mathrm{eff}}(\overrightarrow{0}, t ; \overrightarrow{0}, 0)$ (with color index $=2$, and Dirac index = 1) from the origin to any point along $t$-axis, for one configuration, at $m_{q} a=m_{b} a=0.68$. (b) Similar to (a), but averaging over all elements with different color and Dirac indices.

for scalar $(S)$, pseudoscalar $(P)$, vector $(V)$, axial-vector $(A)$, and tensor $(T)$ mesons, with Dirac matrix $\Gamma=\left\{\mathbb{I}, \gamma_{5}, \gamma_{i}, \gamma_{5} \gamma_{i}, \gamma_{5} \gamma_{4} \gamma_{i}\right\}$ respectively, for both symmetric $\left(m_{Q}=m_{q}\right)$ and asymmetric (fixed $m_{Q}$ with various $m_{q}$ ) quark masses. Finally we extract their masses and pseudoscalar decay constants, compare our results with the experimental data, and also make some theoretical predictions.

\section{Determination of $a^{-1}, m_{c}, m_{s}$, and $m_{b}$}

In Ref. [5], we determine the inverse lattice spacing from the pion decay constant, with the experimental input $f_{\pi}=131 \mathrm{MeV}$. However, in this work, our smallest quark mass turns out to be too heavy (about $m_{s} / 2$ ), thus the chiral extrapolation to $m_{q} \simeq 0$ does not seem to be feasible. Instead, we use the mass and decay constant of the pseudoscalar meson $\eta_{c}(2980)$ to determine the bare mass of charm quark $m_{c}$ and $a^{-1}$ simultaneously, which can be seen in the following.

We measure the time-corelation function Eq.(1.1) of the pseudoscalar meson $C_{P}(t)\left(\Gamma=\gamma_{5}\right)$ for symmetric quark mass $m_{Q}=m_{q}$, and fit it to the usual formula $G(t)$, to extract the meson mass $m_{P} a$ and the decay constant $f_{P} a$ :

$$
G(t)=\frac{z^{2}}{2 m_{P} a}\left[e^{-m_{P} a t}+e^{-m_{P} a(T-t)}\right], \quad f_{P} a=2 m_{q} a \frac{z}{m_{P}^{2} a^{2}}
$$

Thus, we obtain the ratio of $m_{P} / f_{P}$ for each $m_{q} a$ we have computed, which can be compared with the ratio of mass and decay constant of $\eta_{c}$.

Although only the $m_{\eta_{c}}$ is measured in the high energy experiments, we can obtain the theoretical value of $m_{\eta_{c}} / f_{\eta_{c}}=6.8(2)$ from our previous study of pseudoscalar mesons on the $20^{3} \times 40$ 


\begin{tabular}{c|cccc}
\hline$m_{q}$ & $\begin{array}{c}\text { lattice } \\
\text { bare mass }\end{array}$ & $\begin{array}{c}\overline{\mathrm{MS}} \\
(\text { at } \mu=2 \mathrm{GeV})\end{array}$ & $\begin{array}{c}\overline{\mathrm{MS}} \\
\left(\text { at } \mu=m_{q}\right)\end{array}$ & $\begin{array}{c}\text { PDG } \\
\text { average }\end{array}$ \\
\hline$m_{b}$ & $5.22(4) \mathrm{GeV}$ & $4.81(5) \mathrm{GeV}$ & $4.65(5) \mathrm{GeV}$ & $4.20(7) \mathrm{GeV}$ \\
$m_{c}$ & $1.23(4) \mathrm{GeV}$ & $1.13(4) \mathrm{GeV}$ & $1.16(4) \mathrm{GeV}$ & $1.25(9) \mathrm{GeV}$ \\
\hline
\end{tabular}

Table 1: The bare mass $\left(m_{q} a^{-1}\right)$ of $\mathbf{b}$ and $\mathbf{c}$ quarks, and their masses in $\overline{\mathrm{MS}}$ scheme at scales $\mu=2 \mathrm{GeV}$ and $\mu=m_{q}$, respectively, in comparison with the PDG average in the last column.

lattice at $\beta=6.1$ [5]. We find that the closest value of $m_{P} / f_{P}=6.8(1)$ occurs at $m_{q} a=0.16$, thus we fix $m_{c} a=0.16$. Further, using the experimental input $m_{\eta_{c}}=2980 \mathrm{MeV}$, we determine the inverse of the lattice spacing $a^{-1}=7680$ (59) MeV. To check the goodness of our determination, we measure the time-correlation function of the vector meson $\overline{\mathbf{c}} \gamma_{i} \mathbf{c}$, and extract its mass equal to 3091(11) MeV, which is in good agreement with $J / \Psi(3097)$.

Next, we determine the bare masses of strange and bottom quark, $m_{s} a$ and $m_{b} a$, by extracting the mass of vector meson from the time-correlation function $C_{V}(t)$ for various $m_{q} a$ we have computed. At $m_{q} a=0.02$, we obtain $m_{V}=1027$ (38) MeV, in good agreement with $\phi(1020)$; while at $m_{q} a=0.68$, we obtain $m_{V}=9453(3) \mathrm{MeV}$, in good agreement with $\Gamma(9460)$. Thus we determine $m_{s} a=0.02$ and $m_{b} a=0.68$, respectively.

Note that although the spatial size of our lattice $(L \simeq 0.8 \mathrm{fm})$ seems to be small, the finite size effects should be well under control since our pseudoscalar meson masses satisfies $m_{P} L>4$ even for our smallest bare quark mass $m_{q} a=0.01$.

In order to compare our results of $m_{b}$ and $m_{c}$ with the high energy phenomenology, we have to obtain the lattice renormalization constants $Z_{m}=Z_{s}^{-1}$ and transcribe them to the usual $\overline{\mathrm{MS}}$ scheme, where $Z_{s}$ is the renormalization constant for $\psi \bar{\psi}$. In general, the renormalization constants should be determined nonperturbatively. However, in this work, the lattice spacing is rather small ( $a \simeq$ $0.026 \mathrm{fm})$, we suspect that the one-loop perturbation formula [6]

$$
Z_{s}(\mu)=1+\frac{g^{2}}{4 \pi^{2}}\left[\ln \left(a^{2} \mu^{2}\right)+0.17154\right], \quad\left(m_{0}=1.30\right)
$$

already provides a very good approximation for $Z_{s}$. At $\beta=7.2, a^{-1}=7.680(59) \mathrm{GeV}$, our results for $m_{b}^{\overline{\mathrm{MS}}}$ and $m_{c}^{\overline{\mathrm{MS}}}$ at scales $\mu=2 \mathrm{GeV}$ and their masses $\left(\mu=m_{q}\right)$ are listed in Table 1. Comparing with the PDG [7] average, our result of $m_{b}^{\overline{\mathrm{MS}}}\left(m_{b}\right)$ turns out to be a little higher, but $m_{c}^{\overline{\mathrm{MS}}}\left(m_{c}\right)$ is in good agreement with the PDG average.

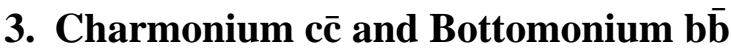

First of all, we check to what extent we can reproduce the charmonium mass spectra which have been measured precisely by high energy experiments. Our results of the mass spectra of the lowest-lying states of charmonium are summarized in Table 2. The first column is the gamma matrix we use to compute the meson time-correlation function (Eq.(1.1). The second and the third columns are the $J^{P C}$ of the state and the conventional spectrascopic notation. The fourth column is the time range for fitting the time-correlation function to the formula in Eq.(2.1). The $\chi^{2} /$ dof is listed in the fifth column, and the extracted meson mass is listed in the sixth column, where the 


\begin{tabular}{lllllll}
\hline$\Gamma$ & $J^{P C}$ & $\mathrm{n}^{2 S+1} L_{J}$ & {$\left[t_{\min }, t_{\max }\right]$} & $\chi^{2} /$ dof & Mass $(\mathrm{MeV})$ & PDG \\
\hline $\mathbb{I}$ & $0^{++}$ & $1^{3} P_{0}$ & {$[19,42]$} & 0.32 & $3413(14)(9)$ & $\chi_{c 0}(3415)$ \\
$\gamma_{5}$ & $0^{-+}$ & $1^{1} S_{0}$ & {$[22,38]$} & 1.02 & $2980(10)(12)$ & $\eta_{c}(2980)$ \\
$\gamma_{i}$ & $1^{--}$ & $1^{3} S_{1}$ & {$[19,38]$} & 1.18 & $3091(11)(14)$ & $J / \psi(3097)$ \\
$\gamma_{5} \gamma_{i}$ & $1^{++}$ & $1^{3} P_{1}$ & {$[18,42]$} & 0.51 & $3516(13)(8)$ & $\chi_{c 1}(3510)$ \\
$\gamma_{5} \gamma_{4} \gamma_{i}$ & $1^{+-}$ & $1^{1} P_{1}$ & {$[19,43]$} & 0.41 & $3526(13)(9)$ & $h_{c}(3524)$ \\
\hline
\end{tabular}

Table 2: The mass spectra of the lowest-lying $(n=1)$ charmonium $\overline{\mathbf{c}} \Gamma \mathbf{c}$ states obtained in this work, in comparison with the PDG values in the last column.

\begin{tabular}{lllllll}
\hline$\Gamma$ & $J^{P C}$ & $\mathrm{n}^{2 S+1} L_{J}$ & {$\left[t_{\min }, t_{\max }\right]$} & $\chi^{2} /$ dof & Mass(MeV) & PDG \\
\hline $\mathbb{I}$ & $0^{++}$ & $1^{3} P_{0}$ & {$[21,39]$} & 0.21 & $9863(15)(8)$ & $\chi_{b 0}(9859)$ \\
$\gamma_{5}$ & $0^{-+}$ & $1^{1} S_{0}$ & {$[27,35]$} & 0.72 & $9383(4)(2)$ & $\eta_{b}(9300) ?$ \\
$\gamma_{i}$ & $1^{--}$ & $1^{3} S_{1}$ & {$[20,39]$} & 1.19 & $9453(3)(2)$ & $\Upsilon(9460)$ \\
$\gamma_{5} \gamma_{i}$ & $1^{++}$ & $1^{3} P_{1}$ & {$[22,38]$} & 0.13 & $9896(20)(8)$ & $\chi_{b 1}(9893)$ \\
$\gamma_{5} \gamma_{4} \gamma_{i}$ & $1^{+-}$ & $1^{1} P_{1}$ & {$[22,38]$} & 0.10 & $9916(22)(8)$ & \\
\hline
\end{tabular}

Table 3: The mass spectra of the lowest-lying $(n=1)$ bottomonium $\overline{\mathbf{b}} \Gamma \mathbf{b}$ states obtained in this work. The last column is the experimental state we have identified, and its PDG mass value.

first error is the statistical error, and the second error is the estimated systematic error based on all fittings satisfying $\chi^{2} /$ dof $<1.3$ and $\left|t_{\max }-t_{\min }\right| \geq 6$, with $t_{\min } \geq 10$ and $t_{\max } \leq 50$. The last column is the corresponding state in high energy experiments, with the PDG mass value. Evidently, our results are in good agreement with the PDG values. Note that our result of the hyperfine splitting $\left(1^{3} S_{1}-1^{1} S_{0}\right)$ is $111(14)(18) \mathrm{MeV}$, comparing with the PDG value $118 \mathrm{MeV}$.

For the pseudoscalar $\eta_{c}$, we also obtain its decay constant $f_{\eta_{c}}$ together with its mass through Eq.(2.1). Our result is $f_{\eta_{c}}=438 \pm 5 \pm 6 \mathrm{MeV}$, where the first error is the statistical error, and the second error is the estimated systematic error, as described previously.

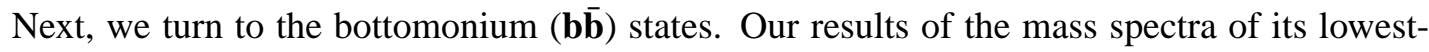
lying states are summarized in Table 3 . For the pseudoscalar $\eta_{b}$, it was first reported by ALEPH Collaboration [8], but it has not been confirmed by other HEP experimental groups. Our theoretical values turns to be a little deviate from their experimental result. In addition, we also determined its decay constant $f_{\eta_{b}}=801 \pm 7 \pm 5 \mathrm{MeV}$.

Finally, we note that the tensor meson $h_{b}$ has not been observed in high energy experiments, thus our result of its mass serves as the first prediction from lattice QCD with exact chiral symmetry.

\section{Mesons with quark contents $\mathbf{s} \bar{b}$ and $\mathbf{c} \bar{b}$}

The unitarity of CKM matrix is one of the crucial tests for the Standard Model. In order to extract the CKM matrix elements, the decay constants of heavy-light pseudoscalar mesons (e.g., $f_{B}, f_{B_{s}}, f_{D}$, and $f_{D_{s}}$ ) have to be determined precisely. In Ref. [5], we have presented our theoretical results of $f_{D}$ and $f_{D_{s}}$ from lattice QCD with exact symmetry, which are in good agreement with the recent experimental results [9] from CLEO Collaboration. 


\begin{tabular}{lllllll}
\hline$\Gamma$ & $J^{P}$ & $\mathrm{n}^{2 S+1} L_{J}$ & {$\left[t_{\min }, t_{\max }\right]$} & $\chi^{2} /$ dof & Mass(MeV) & PDG \\
\hline $\mathbb{I}$ & $0^{+}$ & $1^{3} P_{0}$ & {$[20,40]$} & 0.38 & $5852(15)(12)$ & $B_{s J}^{*}(5850)$ \\
$\gamma_{5}$ & $0^{-}$ & $1^{1} S_{0}$ & {$[26,32]$} & 0.56 & $5385(27)(17)$ & $B_{s}(5368)$ \\
$\gamma_{i}$ & $1^{-}$ & $1^{3} S_{1}$ & {$[25,33]$} & 0.50 & $5424(28)(19)$ & $B_{s}^{*}(5412)$ \\
$\gamma_{5} \gamma_{i}$ & $1^{+}$ & $1^{3} P_{1}$ & {$[18,42]$} & 0.68 & $5884(16)(13)$ & \\
$\gamma_{5} \gamma_{4} \gamma_{i}$ & $1^{+}$ & $1^{1} P_{1}$ & {$[18,38]$} & 0.62 & $5897(16)(12)$ & \\
\hline
\end{tabular}

Table 4: The mass spectra of the lowest-lying $\overline{\mathbf{b}} \Gamma \mathbf{s}$ meson states obtained in this work. The last column is the experimental state we have identified, and its PDG mass value.

\begin{tabular}{lllllll}
\hline$\Gamma$ & $J^{P}$ & $\mathrm{n}^{2 S+1} L_{J}$ & {$\left[t_{\min }, t_{\max }\right]$} & $\chi^{2} /$ dof & $\operatorname{Mass}(\mathrm{MeV})$ & PDG \\
\hline $\mathbb{I}$ & $0^{+}$ & $1{ }^{3} P_{0}$ & {$[19,41]$} & 0.26 & $6732(13)(9)$ & \\
$\gamma_{5}$ & $0^{-}$ & $1{ }^{1} S_{0}$ & {$[19,38]$} & 1.14 & $6278(6)(4)$ & $B_{c}(6286)$ \\
$\gamma_{i}$ & $1^{-}$ & $1{ }^{3} S_{1}$ & {$[19,38]$} & 1.31 & $6315(6)(5)$ & \\
$\gamma_{5} \gamma_{i}$ & $1^{+}$ & $1{ }^{3} P_{1}$ & {$[19,41]$} & 0.27 & $6778(12)(7)$ & \\
$\gamma_{5} \gamma_{4} \gamma_{i}$ & $1^{+}$ & $1{ }^{1} P_{1}$ & {$[18,42]$} & 0.45 & $6796(10)(7)$ & \\
\hline
\end{tabular}

Table 5: The mass spectra of the lowest-lying $\overline{\mathbf{b}} \Gamma \mathbf{c}$ meson states obtained in this work. The last column is the experimental state we have identified, and its PDG mass value.

In this work, we extract the masses and the decay constants of pseudoscalar mesons with $\mathbf{s} \overline{\mathbf{b}}$ and $\mathbf{c} \overline{\mathbf{b}}$, via fitting their time-correlation functions $C_{P}(t)$ to the formula

$$
F(t)=\frac{z^{2}}{2 m_{P} a}\left[e^{-m_{P} a t}+e^{-m_{P} a(T-t)}\right], \quad f_{P} a=\left(m_{q}+m_{Q}\right) a \frac{z}{m_{P}^{2} a^{2}}
$$

Our results for $B_{s}$ are $m_{B_{s}}=5385(27)(17) \mathrm{MeV}$, and $f_{B_{s}}=253(8)(7) \mathrm{MeV}$. The former is in good agreement with the PDG value $(5368 \mathrm{MeV})$. The later has not been measured in HEP experiments yet, thus our result serves as the first prediciton from lattice QCD with exact chiral symmetry.

In Table 4, we present our results of the mass spectra of the lowest-lying states of beauty mesons with quark content $\mathbf{s} \overline{\mathbf{b}}$. Here we have identified the scalar $\overline{\mathbf{b}} \mathbf{s}$ meson with the state $B_{S J}^{*}(5850)$ observed in HEP experiments, due to the proximity of their masses. Theoretically, this implies that $B_{S J}^{*}(5850)$ possesses $J^{P}=0^{+}$, which can be verified by HEP experiments in the future. Further, our results of the masses of the axial-vector and tensor mesons, which have not been observed experimentally, serves a prediction from lattice QCD.

For the pseudoscalar meson $B_{c}$, we obtain $m_{B_{c}}=6278(6)(4) \mathrm{MeV}$, and $f_{B_{c}}=489(4)(3) \mathrm{MeV}$. Our result of $m_{B_{c}}$ is in good agreement with the experimental value 6286(5) MeV measured by CDF Collaboration [10], but $f_{B_{c}}$ has not been determined in HEP experiments. In principle, $f_{B_{c}}$ can be measured from the leptonic decay $B_{c}^{+} \rightarrow l^{+} v_{l}$, since its decay width is proportional to $f_{B_{c}}^{2}\left|V_{c b}\right|^{2}$.

In Table 5, we summarize our results of the mass spectra of the lowest-lying states of mesons with beauty and charm. Except for the pseudoscalar $B_{c}$, other states have not been observed in experiments. It will be interesting to see to what extent the experimental results would agree with our theoretical values. 


\begin{tabular}{clllll}
$\overline{\mathbf{Q}} \Gamma q$ & {$\left[t_{\min }, t_{\max }\right]$} & $\chi^{2} /$ dof & Mass $(\mathrm{MeV})$ & $f_{P}(\mathrm{MeV})$ & PDG \\
$\overline{\overline{\mathbf{b}}} \gamma_{5} b$ & {$[27,35]$} & 0.72 & $9383(4)(2)$ & $801(7)(5)$ & $\eta_{b}(9300)$ \\
$\overline{\mathbf{b}} \gamma_{5} c$ & {$[19,38]$} & 1.14 & $6278(6)(4)$ & $489(4)(3)$ & $B_{c}(6287)$ \\
$\overline{\mathbf{b}} \gamma_{5} s$ & {$[26,32]$} & 0.56 & $5385(27)(17)$ & $253(8)(7)$ & $B_{s}(5368)$ \\
$\overline{\mathbf{c}} \gamma_{5} c$ & {$[22,38]$} & 1.02 & $2980(10)(12)$ & $438(5)(6)$ & $\eta_{c}(2980)$ \\
\hline
\end{tabular}

Table 6: The decay constants of pseudoscalar mesons obtained in this work, together with their masses. They are identified with the corresponding PDG mesons listed in the last column.

\section{Concluding remarks}

We have performed the first study of treating $\mathbf{b}, \mathbf{c}$ and $\mathbf{s}$ quarks as Dirac fermions in lattice QCD with exact chiral symmetry. The lowest-lying mass spectra of mesons with quark contents $\mathbf{b} \overline{\mathbf{b}}, \mathbf{c} \overline{\mathbf{c}}$, $\mathbf{s} \overline{\mathbf{b}}$, and $\mathbf{c} \overline{\mathbf{b}}$ are determined, together with the pseudoscalar decay constants (which is summarized in Table 6). Furthermore, the $\mathbf{b}$ and $\mathbf{c}$ quark masses are determined (see Table 1). Our results suggest that lattice QCD with exact chiral symmetry is a viable approach to study heavy quark physics from the first principles of QCD.

For systems with $\mathbf{u}$ / d quarks, one may use several quark masses in the range $m_{u / d}<m_{q}<m_{s}$ to perform the chiral extrapolation. With a coarser and larger lattice, say, $42^{3} \times 64$ at $\beta=7.0$, it is possible to accomodate a wider range of quark masses $m_{s} / 4<m_{q}<m_{b}$. This study is now in progress.

\section{Acknowledgement}

This work was supported in part by the National Science Council, Republic of China, under the Grant No. NSC95-2112-M002-005 (T.W.C.), and Grant No. NSC95-2112-M001-072 (T.H.H.), and by the National Center for High Performance Computation, and the Computer Center at National Taiwan University.

\section{References}

[1] T. W. Chiu, T. H. Hsieh, C. H. Huang, and K. Ogawa [TWQCD Collaboration], Phys. Lett. B 651, 171 (2007).

[2] T. W. Chiu and T. H. Hsieh [TWQCD Collaboration], PoS LAT2006, 180 (2007)

[3] T. W. Chiu, Phys. Rev. Lett. 90, 071601 (2003);

[4] T. W. Chiu, hep-lat/0303008; Nucl. Phys. Proc. Suppl. 129, 135 (2004).

[5] T. W. Chiu, T. H. Hsieh, J. Y. Lee, P. H. Liu and H. J. Chang, Phys. Lett. B 624, 31 (2005)

[6] C. Alexandrou, E. Follana, H. Panagopoulos and E. Vicari, Nucl. Phys. B 580, 394 (2000).

[7] W. M. Yao et al. [Particle Data Group], J. Phys. G 33 (2006) 1.

[8] A. Heister et al. [ALEPH Collaboration], Phys. Lett. B 530, 56 (2002)

[9] M. Artuso et al. [CLEO Collaboration], Phys. Rev. Lett. 95, 251801 (2005); Phys. Rev. Lett. 99, 071802 (2007).

[10] A. Abulencia et al. [CDF Collaboration], Phys. Rev. Lett. 96, 082002 (2006) 